\title{
Factors Favoring Long Term Survival in Patients with Stage IV Epithelial Ovarian Cancer: An Institutional Research
}

\author{
Hafiz Abubakar Sarwar', Jhanzeb Iftikhar'1, Samia Yasmeen', Fareeha Sheikh', \\ Fatima Ali ${ }^{1}$, Aamir Ali Syed ${ }^{2}$, Neelam Siddiqui ${ }^{1}$
}

'Department of Medical Oncology, Shaukat Khanum Memorial Cancer Hospital and Research Center, Lahore, Pakistan. ${ }^{2}$ Department of Surgical Oncology, Shaukat Khanum Memorial Cancer Hospital and Research Center, Lahore, Pakistan.

\begin{abstract}
Objective: Our aim was to identify factors favoring long term survival in patients presenting with stage IV epithelial ovarian cancer. Methods: We did retrospective analysis of thirty patients with stage IV epithelial ovarian cancer diagnosed and treated at Shaukat Khanum Memorial Cancer Hospital, Lahore, Pakistan from 2006 to 2013. Patient's demographics, clinical data and histopathology were abstracted from cancer registry department of our hospital. Chi-square test was used to find the association between clinic-pathological variables and long term survival. Result: All patients received chemotherapy and surgery as per ovarian cancer guidelines. Of the thirty patients, eleven patients survived greater than four years median survival was recorded as thirty five months. Absence of co-morbidities and good performance status indicated good results of therapy however did not have statistically significant impact on survival. Higher CA-125 at presentation i.e. $>1000$ (normal range: $<21 \mathrm{U} / \mathrm{ml}$ ), response to initial chemotherapy, interval cytoreductive surgery and complete response after induction therapy were significantly associated with long term survival $(\mathrm{P}<0.05)$. Conclusion: Prognosis of patients presenting with stage IV epithelial ovarian cancer remains poor. Very high values of CA-125 (>1000) at presentation, response to initial chemotherapy, interval surgical resection and complete remission after induction therapy, appear to be significant prognostic factors for long term survival. Further studies exploring molecular profiling and immunological factors are warranted.
\end{abstract}

Keywords: Ovarian Cancer- stage IV- prognostic factor- survival

Asian Pac J Cancer Care, 5 (1), 15-18

\section{Introduction}

Ovarian cancer accounts for 3\% of all cancers among women and is the 5 th leading cause of cancer related death world wide [1]. Epithelial ovarian cancer (EOC) accounts for $90 \%$ of all malignant tumors of ovary and these are adenocarcinomas. EOC are further classified into Type I and Type II tumors [2-3]. Type I tumors are considered low grade and include endometrioid, mucinous and clear cell histologies and they carry mutations of BRAF, KRAS and PTEN with microsatellite instability. While type II tumors are high grade serous and carcinosarcomas which harbor mutations in BRCA1, BRCA2 and p53 [4].

Ovarian cancer usually presents in postmenopausal women with history of abdominal pain and distension. Seventeen percent of the patients present with stage
Submission Date: 11/05/2019 Acceptance Date: 01/05/2020

IV disease, where 5-years survival rate remains less than 10\% [5-6]. Currently with advanced treatment modalities, a significant number of patients achieve complete biochemical and radiological response. However patients with stage IV disease tend to relapse within eighteen months after treatment completion [3]. Among the relapsed patients, in whom tumor remains sensitive to platinum based chemotherapy, outcomes are good as compared to those whose disease becomes platinum refractory in subsequent relapses [3].

Approximately 295,000 women developed ovarian cancer every year world wide out of which $90 \%$ are epithelial ovarian cancers [1]. In Pakistan, its incidence is also rising by $1-2 \%$ every year and various studies conducted in Pakistan report it as among the ten most common cancers in Pakistan [7-8]. Cress RD et al.,

Corresponding Author:

Dr. Hafiz Abubakar Sarwar

Department of Medical Oncology, Shaukat Khanum memorial cancer hospital and Research center, Lahore, Pakistan.

Email: dr.bakar160@gmail.com 
evaluated characteristics of long term survival in 3,582 patients of ovarian cancer at all stages of presentation and found that younger age, early stage, low grade and non-serous histology are predictors of long term survival, but some women with high risk cancer lived longer [9]. Hamilton CA et al., conducted a study on 3010 patients with advanced stage epithelial ovarian cancer and found that 195 patients survived greater than 10 years. Out of these patients, the number of stage IV patient were very low and the outcomes were worse. They revealed that lower CA-125 value, absence of ascites, stage at presentation and $\mathrm{R} 0$ resection had significant influence on long term survival [10]. It is observed that there is no significant survival data available for Pakistani population due to lack of studies. Secondly majority of existing literature shows combined data of stage III and stage IV so we specifically studied stage IV patients in this study.

\section{Materials and Methods}

The present time based research was conducted in SKMCH\&RC. All patients who presented with stage IV epithelial ovarian cancer from 2006 to 2013 were included. Thirty patients with newly diagnosed stage IV EOC were analyzed. Patient's baseline characteristics including age, comorbidities, ECOG-performance status and BMI were recorded. Details of therapy including surgical procedure underwent obtained. All of the patients received neoadjuvant platinum containing regimens as per ovarian cancer protocol. CA-125 was recorded at baseline, after completion of treatment and on subsequent follow-ups. The histological type, site of metastasis and ascites were also recorded.

\section{Statistical analysis}

SPSS software (version 23.0; SPSS, Chicago, IL, USA) was used for statistical analysis of the data. Mean \pm standard deviation was used for continuous variables while frequencies and percentages used for categorical variables. Chi square test or fisher's exact test (when necessary) were used to compare categorical variable. The Kaplan-Meier method was used to estimate survival as a function of time, and survival differences were analyzed by the log-rank test. Statistical significance was defined as a two-tailed p-value 0.05. Approval for study was taken from our Institutional Review Board (IRB).

\section{Results}

Table 1 presents the demographic and clinical characteristics of 30 stage IV ovarian cancer patients with a mean age and standard deviation of $50 \pm 13$ years. All the patients were females with mean body mass index and standard deviation of $27 \pm 6.0$. In addition, more than a half (57\%) of the respondents had no comorbidity and $(23 \%)$ had multiple comorbidities. Additionally, majority (63\%) of the patients were died on last follow-up and the median survival was survival was found thirty five months. Among patients who survived, half of them had ECOG performance status of 2 (meaning that patients were ambulatory and capable of all self-care but unable to carry out any work activities; up and about more than $50 \%$ of waking hours).

Stratification of age, body mass index, comorbidity and ECOG performance status with respect to status (alive and death) is shown in Table 1.

Furthermore, Table 2 presents the pathological characteristics of patients with an overall mean CA-125 value and standard deviation of $4100 \pm 954$. Majority of the patients $(63 \%)$ had serous histopathology and more than half $(53 \%)$ of the patients had M-1 site of metastasis. Moreover, surgical procedure was performed in (70\%) of the patients and more than half of patients showed complete response to chemotherapy as shown in Table 2 . Therefore,

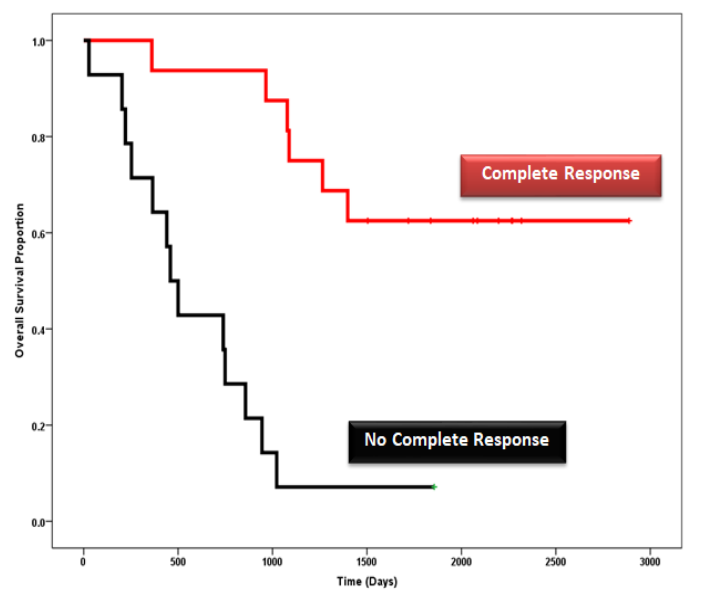

Figure 1. Overall Survival was Compared between Complete Response and no Complete Response

Table 1. Descriptive Statistics (Demographics and Clinical characteristics)

\begin{tabular}{llcccc}
\hline Variables & Categories & Total = N (\%) & Alive 11 (36.7\%) & Death 19 (63.3\%) & p-value \\
\hline Age (years) & Mean \pm SD* $^{*}$ & $49.97 \pm 12.95$ & $48.55 \pm 6.96$ & $50.79 \pm 15.53$ & 0.66 \\
Body Mass Index & Mean $\pm \mathrm{SD}^{*}$ & $27.33 \pm 5.70$ & $26.89 \pm 5.34$ & $27.58 \pm 6.03$ & 0.75 \\
Comorbids & No & $17(56.7 \%)$ & $6(35.3 \%)$ & $11(64.7 \%)$ & 0.71 \\
& Single & $6(20.0 \%)$ & $3(50.0 \%)$ & $3(50.0 \%)$ & \\
& Multiple & $7(23.3 \%)$ & $2(28.6 \%)$ & $5(71.4 \%)$ & \\
ECOG-PS & $1^{* *}$ & $12(40.0 \%)$ & $5(41.7 \%)$ & $7(58.3 \%)$ & 0.38 \\
& $2^{* *}$ & $15(50.0 \%)$ & $6(40.0 \%)$ & $9(60.0 \%)$ & \\
& $3^{* *}$ & $3(10.0 \%)$ & $0(0.0 \%)$ & $3(100.0 \%)$ & \\
\hline
\end{tabular}

*standard deviation; $1 * *$, Ambulatory; $2 * *$, In bed $<50 \% ; 3 * *$, In bed $>50 \%$. 
Table 2. Descriptive Statistics (Pathological Characteristics)

\begin{tabular}{|c|c|c|c|c|c|}
\hline Variables & Categories & $N(\%)$ & Alive $11(36.7 \%)$ & Death $19(63.3 \%)$ & p-value \\
\hline CA-125 & Mean $\pm \mathrm{SE}^{*}$ & $4100.17 \pm 954.38$ & $5165.10 \pm 1557.34$ & $3425.32 \pm 1211.62$ & 0.36 \\
\hline \multirow[t]{4}{*}{ Histopathology } & Adeno unspecified & $3(10.0 \%)$ & $0(0.0 \%)$ & $3(100.0 \%)$ & 0.64 \\
\hline & Serous & $19(63.3 \%)$ & $8(42.1 \%)$ & $11(57.9 \%)$ & \\
\hline & Mucinous & $2(6.7 \%)$ & $1(50.0 \%)$ & $1(50.0 \%)$ & \\
\hline & Others & $6(20.0 \%)$ & $2(33.3 \%)$ & $4(66.7 \%)$ & \\
\hline \multirow[t]{2}{*}{ Site of Metastasis } & M-1 & $16(53.3 \%)$ & $5(31.2 \%)$ & $11(68.8 \%)$ & 0.71 \\
\hline & M-2 & $14(46.7 \%)$ & $6(42.9 \%)$ & $8(57.1 \%)$ & \\
\hline \multirow[t]{2}{*}{ Ascites } & Yes & $26(84.7 \%)$ & $9(34.6 \%)$ & $17(65.4 \%)$ & 0.61 \\
\hline & No & $4(13.3 \%)$ & $2(50.0 \%)$ & $2(50.0 \%)$ & \\
\hline Number of Chemotherapy cycles & Mean $\pm \mathrm{SD}^{* *}$ & $22.0 \pm 21.0$ & $34.91 \pm 28.61$ & $14.47 \pm 10.20$ & 0.01 \\
\hline \multirow[t]{2}{*}{ Surgical Resection } & Surgery Performed & $21(70.0 \%)$ & $8(38.1 \%)$ & $13(61.9 \%)$ & 1.00 \\
\hline & No surgery performed & $9(30.0 \%)$ & $3(33.3 \%)$ & $6(66.7 \%)$ & \\
\hline \multirow[t]{2}{*}{ Response } & Complete Response & $16(53.3 \%)$ & $10(62.5 \%)$ & $6(37.5 \%)$ & 0.002 \\
\hline & No Complete Response & $14(46.7 \%)$ & $1(7.1 \%)$ & $13(92.9 \%)$ & \\
\hline Survival Time (days) & Mean \pm SD & $1197 \pm 789.94$ & $2091.10 \pm 111.36$ & $680.80 \pm 91.69$ & 0.001 \\
\hline
\end{tabular}

*standard error; ** standard deviation

overall survival was found statistically significant (p-value 0.002 using chi-square test) among patients who showed complete response to initial therapy as shown in Table 2. Overall survival was compared between complete response and no complete response. There was statistically significant difference ( $\mathrm{p}=0.001 \mathrm{using}$ independent sample t-test) between the two groups as shown in Figure 1.

\section{Discussion}

In our study, $70 \%$ stage IV ovarian cancer patients received neo-adjuvant platinum based chemotherapy followed by surgery as per ovarian cancer guidelines. Among thirty patient twenty one (70\%) patients underwent cytoreductive surgery while nine patients $(30 \%)$ were not fit enough to undergo surgery. The outcomes were comparable for both groups in terms of long term survival. [10] Hamilton CA et al, similar results describing that cytoreductive surgery did not influence the outcomes and $15 \%$ of patients survived more than 10 years. Surprising results were found in a study performed by Cress RD et al., that one third of patients with stage III and IV disease and serous histology showed long term survival of more than 10 years. The possible reason for this finding was improved surgical techniques with no or minimal residual disease and concomitant use of intraperitoneal and adjuvant chemotherapy and the possibility of somatic mutations in patients with same histology [9].

A contrasting result was found regarding CA-125 level values. In our study the impact of CA-125 levels acts differently. High values of CA-125 at presentation were linked to long term survival as compared to previous studies, though it was not statistically significant $(\mathrm{p}=0.36)$. The reason for this finding might be ethnic variation in our region. Existing literature showed that lower pretreatment CA-125 values is associated with long term survival [11].

Tumor histology is also an important predictor of survival [12-14]. In our study population, the most common histological type was serous (63\%) and remaining histologies were mucinous (7\%), adeno-unspecified (10\%) and others were (20\%). None of the histological subtype was associated with statistically significant long term survival ( $p$-value $=0.64)$.

In sixteen patients (53\%) the primary site of metastasis was pleural effusion (M1) while in fourteen patients (47\%) metastatic sites were other than pleura (M2). Among M2 metastasis six patients (43\%) had better long term survival of more than four years. In this study, twenty six $(85 \%)$ patients had ascites at the time of presentation while four patients $(13 \%)$ had no ascites.

It was found that the eleven patients $(37 \%)$ who responded and tolerated neoadjuvant platinum based chemotherapy showed long term survival of more than four years and the results were also statistically significant $(p$-value $=0.01)$ as compared to 19 patients $(63 \%)$ whose disease was not responsive to chemotherapy and could received (14 \pm 10 number of chemotherapy cycles) less number of chemotherapy cycles. The results were also significant. Melamed A, et al,. and Rosen B, et al,. showed that neoadjuvant chemotherapy for advance ovarian cancer has survival benefits [15-16].

One of the most important predictor of long term survival found in this study was achievement of complete radiological and biochemical response after neoadjuvant chemotherapy, surgery and adjuvant chemotherapy $(p$-value $=0.02)$. In this study group $16(53 \%)$ patients achieved complete response. Among them 10 (63\%) patients showed long term survival of more than four years while $6(38 \%)$ patient showed long term survival of less than four years. While 14 (47\%) patients could not achieve a complete response. Among them only one patient (7\%) lived for more than 4 years while $13(93 \%)$ patients survived less than four years. P value (0.002) also showed that there is an association between response status and long term survival.

In conclusion, advanced stage ovarian cancer is still 
a fatal cancer with poor outcomes. Achieving complete response after neoadjuvant chemotherapy, cytoreductive surgery adjuvant chemotherapy has significant prognostic impact on patient's long term survival. Further studies looking for patterns of gene expressions associated with long term survival, molecular profiling and immunological factors are needed.

\section{Acknowledgements}

I am highly indebted to my respected mentor Dr. Neelam Siddiqui, Medical Oncologist at Shaukat Khanum Memorial Cancer Hospital and Research center, Lahore, Pakistan for her valuable guidance, encouragement and constant supervision during the accomplishment of this task.

\section{References}

1. International Agency for Research on Cancer. Global cancer statistics. 2018. http://globocan.iarc.fr/Pages/fact_sheets_ population.aspx (accessed June 6, 2019).

2. Shih IM, Kurman RJ. Ovarian tumorigenesis: a proposed model based on morphological and molecular genetic analysis. The American journal of pathology. 2004 May $1 ; 164(5): 1511-8$.

3. Jayson GC, Kohn EC, Kitchener HC, Ledermann JA. Ovarian cancer. The Lancet. 2014;384(9951):1376-88.

4. Banerjee S, Kaye SB. New strategies in the treatment of ovarian cancer: current clinical perspectives and future potential. Clinical cancer research. 2013;19(5):961-8.

5. McGuire WP, Hoskins WJ, Brady MF, Kucera PR, Partridge EE, Look KY, Clarke-Pearson DL, Davidson M. Cyclophosphamide and cisplatin compared with paclitaxel and cisplatin in patients with stage III and stage IV ovarian cancer. New England Journal of Medicine. 1996;334(1):1-6.

6. Ozols RF, Bundy BN, Greer BE, Fowler JM, Clarke-Pearson D, Burger RA, Mannel RS, DeGeest K, Hartenbach EM, Baergen R. Phase III trial of carboplatin and paclitaxel compared with cisplatin and paclitaxel in patients with optimally resected stage III ovarian cancer: a Gynecologic Oncology Group study. Journal of Clinical Oncology. 2003;21(17):3194-200.

7. Saeed S, Akram M. EPITHELIAL OVARIAN CANCER. The Professional Medical Journal. 2012;19(01):040-5.

8. Bhurgi Y, Bhurgi A, Hassan SH. Cancer incidence in Karachi, Pakistan: first results from Karachi Cancer Registry. Int J Cancer 2000; 85: 325-29.

9. Cress RD, Chen YS, Morris CR, Petersen M, Leiserowitz GS. Characteristics of long-term survivors of epithelial ovarian cancer. Obstetrics and gynecology. 2015;126(3):491.

10. Hamilton CA, Miller A, Casablanca Y, Horowitz NS, Rungruang B, Krivak TC, Richard SD, Rodriguez N, Birrer MJ, Backes FJ, Geller MA. Clinicopathologic characteristics associated with long-term survival in advanced epithelial ovarian cancer: an NRG Oncology/Gynecologic Oncology Group ancillary data study. Gynecologic oncology. 2018;148(2):275-80.

11. Zorn KK, Tian C, McGuire WP, Hoskins WJ, Markman M, Muggia FM, Rose PG, Ozols RF, Spriggs D, Armstrong DK. The prognostic value of pretreatment CA 125 in patients with advanced ovarian carcinoma: a Gynecologic Oncology Group study. Cancer: Interdisciplinary International Journal of the American Cancer Society. 2009;115(5):1028-35.
12. Schnack TH, Høgdall E, Nedergaard L, Høgdall C. Demographic clinical and prognostic factors of primary ovarian adenocarcinomas of serous and clear cell histology A comparative study. IntJ Gynecol Cancer.2016;26:82-90.

13. Chan JK, Teoh D, Hu JM, Shin JY, Osann K, Kapp DS. Do clear cell ovarian carcinomas have poorer Prognosis compared to other epithelial cell types? A study of 1411 clear cell ovarian cancers. Gynecol Oncol. 2008; 109: 370-376.

14. Ye S, Yang J, You Y, Cao D, Huang H, Wu M, etal. Comparison of clinical characteristic and prognosis Between ovarian clear cell carcinoma and serouscarcinoma: A 10year cohort study of Chinese patients. PLoSOne. 2015; 10:e 0133498

15. Melamed A, Fink G, Wright AA, Keating NL, Gockley AA, Del Carmen MG, Schorge JO, Rauh-Hain JA. Effect of adoption of neoadjuvant chemotherapy for advanced ovarian cancer on all cause mortality: quasi-experimental study. BMJ. 2018;360:j5463.

16. Rosen B, Laframboise S, Ferguson S, Dodge J, Bernardini M, Murphy J, Segev Y, Sun P, Narod SA. The impacts of neoadjuvant chemotherapy and of debulking surgery on survival from advanced ovarian cancer. Gynecologic oncology. 2014;134(3):462-7.

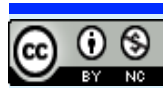

This work is licensed under a Creative Commons AttributionNon Commercial 4.0 International License. 